\title{
Origin of an enhanced colossal magnetoresistance effect in epitaxial $\mathrm{Nd}_{0.52} \mathrm{Sr}_{0.48} \mathrm{MnO}_{3}$ thin films
}

\author{
V. G. Prokhorov ${ }^{\text {a) }}$ and G. G. Kaminsky \\ Institute of Metal Physics, National Academy of Sciences of Ukraine, Kiev 03142, Ukraine \\ J. M. Kim, T. W. Eom, J. S. Park, and Y. P. Lee \\ q-Psi and Department of Physics, Hanyang University, Seoul 133-791, Korea
}

V. L. Svetchnikov

National Center for HREM, TU Delft 2628AL, The Netherlands

G. G. Levtchenko, Yu. M. Nikolaenko, and V. A. Khokhlov

Donetsk Institute for Physics and Technology, National Academy of Sciences of Ukraine, Donetsk 83114, Ukraine

(Submitted June 18, 2010)

Fiz. Nizk. Temp. 37, 392-396 (April 2011)

\begin{abstract}
$\mathrm{Nd}_{0.52} \mathrm{Sr}_{0.48} \mathrm{MnO}_{3}$ films of various thicknesses have been prepared by dc magnetron sputtering on single crystal $\mathrm{LaAlO}_{3}(001)$ substrates. Reducing the film thickness leads to a significant suppression of ferromagnetic (FM) ordering and the Curie point falls below the antiferromagnetic (AFM) transition temperature. When this occurs, a huge rise of the magnetoresistance ratio from 400 to $60000 \%$ is observed in an applied magnetic field of $5 \mathrm{~T}$. We surmise that this new kind of the enhanced colossal magnetoresistance effect originates in the FM/AFM competition and the collapse of the charge-ordered state at high magnetic fields, rather than in the regular double-exchange mechanism. (C) 2011 American Institute of Physics. [doi: 10.1063/1.3592229]
\end{abstract}

\section{INTRODUCTION}

Hole-doped manganites $\mathrm{L}_{1-\mathrm{x}} \mathrm{A}_{x} \mathrm{MnO}_{3}$, where $\mathrm{L}$ and $\mathrm{A}$ are a trivalent lanthanide ion and a divalent alkaline-earth ion, respectively, have attracted considerable attention due to their interesting properties, especially colossal magnetoresistance (CMR), and potential for applications. ${ }^{1}$ The CMR phenomenon is traditionally interpreted in terms of a competition between the double-exchange (DE), which leads to a ferromagnetic (FM) metal state, and the cooperative JahnTeller effect, resulting in polaron localization at temperatures above the Curie point $\left(T_{C}\right)$. However, it has been shown recently that incorporation of the antiferromagnetic (AFM) interaction can be drastically enhance the CMR effect, increasing the magnetoresistance (MR) ratio to as high as $10000 \% .^{2}$ In that case the CMR is associated with the development of short-range correlations among polarons above $T_{C}$, which resemble the charge configuration in the charge-ordered (CO) state, which is stabilized by the AFM interactions. Therefore, experimental observation of an enhanced CMR effect is more to be expected in the manganites with $T_{N} \gtrsim T_{C}$, where $T_{N}$ is the Néel temperature. A $\mathrm{Nd}_{0.52} \mathrm{Sr}_{0.48} \mathrm{MnO}_{3}$ thin film is a more suitable object for a test of this theoretical approach, because FM and AFM phases are present and the transition temperature can be controlled by the deposition mode. ${ }^{3}$ It has also been shown that both phases are formed from the paramagnetic state and exist separately from one other.

In this paper we report experimental results for $\mathrm{Nd}_{0.52} \mathrm{Sr}_{0.48} \mathrm{MnO}_{3}$ films deposited on single crystal $\mathrm{LaAlO}_{3}$ (001) substrates. The origin of the observed enhanced CMR is discussed in detail.

\section{EXPERIMENTAL TECHNIQUES}

The films were prepared by dc magnetron sputtering at a substrate temperature of $650{ }^{\circ} \mathrm{C}$. ${ }^{4}$ Under these conditions the $\mathrm{Nd}_{0.52} \mathrm{Sr}_{0.48} \mathrm{MnO}_{3}$ (NSMO) films with a thickness of $d \simeq 50$, 110 and $160 \mathrm{~nm}$ were deposited on single crystal $\mathrm{LaAlO}_{3}$ (001) (LAO) substrates. $\theta-2 \theta$ x-ray diffraction (XRD) patterns were obtained with a Rigaku diffractometer in $\mathrm{Cu} K_{\alpha}$ radiation. The lattice parameters evaluated directly from the XRD data were plotted against $\cos ^{2} \theta / \sin \theta$. From the intercept of the extrapolated straight line to $\cos ^{2} \theta / \sin \theta=0$, a more precise lattice parameter was obtained. High-resolution electron microscopy (HREM) was carried out using a Philips CM300UT-FEG microscope with a field emission gun operated at $300 \mathrm{kV}$. The point resolution of the microscope was in the order of $0.12 \mathrm{~nm}$. The cross-sectional specimens were prepared by the standard techniques using mechanical polishing followed by ion-beam milling at grazing incidence. All the microstructure measurements were carried out at room temperature. The resistance measurements were made by the four-probe method at temperatures of $4.2-300 \mathrm{~K}$ and in magnetic fields up to $5 \mathrm{~T}$. Field-cooled (FC) and zero-field-cooled (ZFC) magnetization curves were taken with a Quantum Design SQUID magnetometer with an in-plane magnetic field orientation. The magnetization curves for the bare substrates were extracted from the raw experimental data.

\section{MICROSTRUCTURE}

Figure 1(a) shows $\theta-2 \theta$ XRD scans for NSMO films with $d \simeq 50$ and $160 \mathrm{~nm}$. Only the fundamental Bragg peaks of high intensity for the film $(F)$ and the substrate $(\mathrm{S})$ were observed, indicating that the deposition yields a highly $c$-oriented 

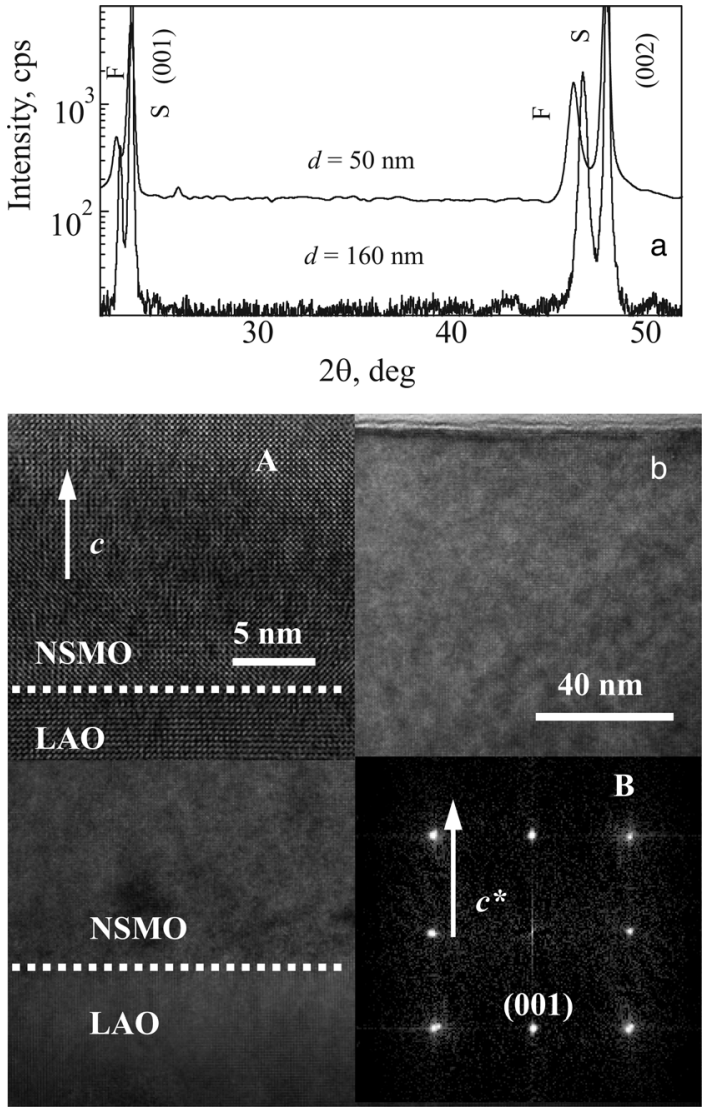

FIG. 1. (a) XRD scans for the NSMO films with different thicknesses. F and $\mathrm{S}$ indicate the fundamental Bragg peaks for the film and the substrate, respectively. (b) A low-magnification cross sectional HREM image taken at room temperature for thick film $(d=160 \mathrm{~nm})$. Inset A is a magnified crosssectional HREM image for the same film. Inset B is the FFT of the HREM image in A. The dashed lines indicate the film/substrate interface, and the white arrows the out-of-plane axis.

crystal structure. This is confirmed by analysis of the transmission contrast of the HREM images. Figure 1(b) shows the cross-sectional HREM image for a thick NSMO film $(d \simeq 160$ $\mathrm{nm})$. Inset A in Fig. 1(b) is a high-magnification view of the same image taken along the [010] zone axis, including the film/substrate interface. It can be seen that the film has an atomically clean and sharp interface without an amorphous intermediate layer or precipitations. The epitaxial relationship for film and substrate was determined to be $[001]_{\mathrm{NSMO}} \|$ $[001]_{\mathrm{LAO}}$. This is confirmed by the corresponding fast Fourier transform (FFT) for the HREM image, shown in inset B of Fig. 1(b), which reveals the almost rectangular pattern of only basic Bragg spots. Microstructure analysis showed that these films have an orthorhombic crystal structure with lattice parameters $a \simeq b \simeq 0.387,0.383$ and $0.379 \mathrm{~nm}$, and $c \simeq 0.388$, 0.39 and $0.394 \mathrm{~nm}$ for $d \simeq 160,110$ and $50 \mathrm{~nm}$, respectively, or very close to published data. ${ }^{5,6}$

Thus, all the deposited films have a homogeneous microstructure without an intermediate layer ${ }^{7}$ and column-like texture. $^{8-10}$ On the other hand, the lattice mismatch between the film and the substrate $(\simeq 2 \%)$ leads to biaxial compressive in-plane and tensile out-of-plane strains.

\section{EXPERIMENTAL RESULTS OF MAGNETIC AND TRANSPORT PROPERTIES}

Figure 2 shows the in-plane FC temperature variations in the magnetic moment, $M(T)$, for NSMO films with thickness

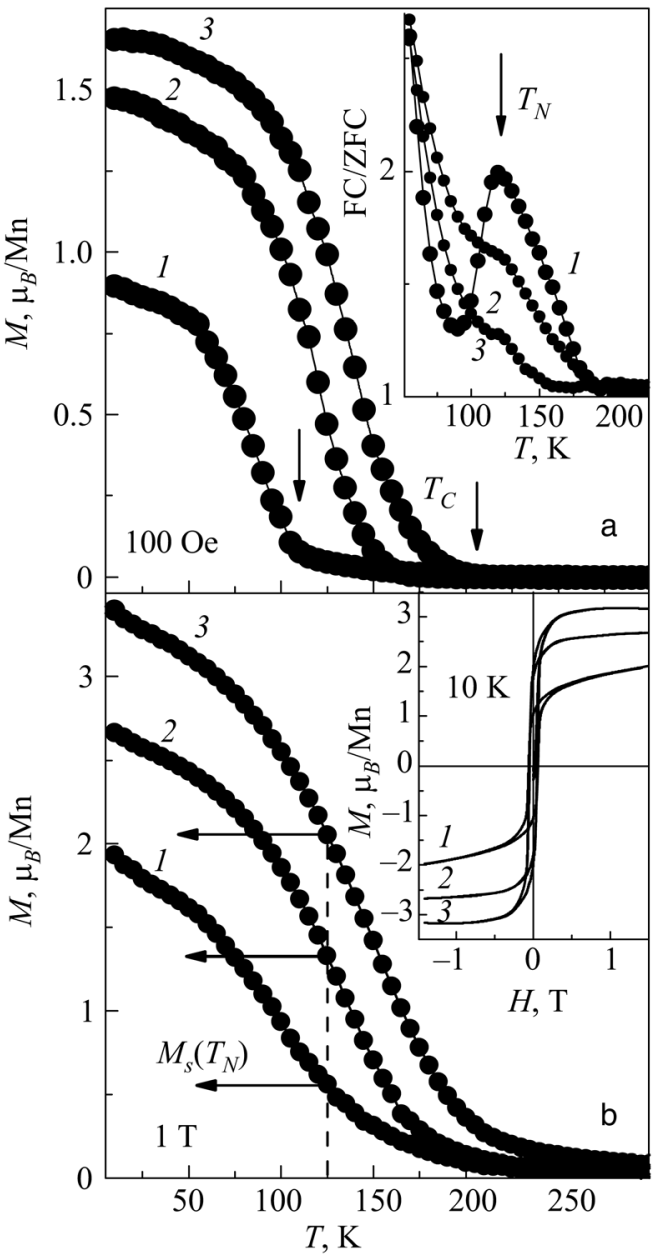

FIG. 2. Temperature dependence of the in-plane FC magnetic moment for NSMO films with thickness $d=50$ (1), 110 (2), and 160 (3) nm in applied magnetic fields of $100 \mathrm{Oe}$ (a) and $1 \mathrm{~T}$ (b). In frame a the arrows indicate the Curie temperature and the inset is the temperature dependence of the reduced FC/ZFC magnetic moment for the same films at an applied magnetic field of 100 Oe. The arrow labelled $T_{N}$ indicates the Néel point. In frame $b$, the dashed line indicates the Néel temperature, the horizontal arrows indicate the saturated magnetic moment at $T_{N}$, and the inset shows the in-plane magnetic hysteresis loops for the same films at $10 \mathrm{~K}$. The curves are only guides for the eye.

$d \simeq 50,110$ and $160 \mathrm{~nm}$ for magnetic fields of 100 Oe and 1 $\mathrm{T}$. The low-field FC $M(T)$ curves reveal a significant thickness dependence of the Curie temperature: $T_{C} \simeq 220,180$ and 115 $\mathrm{K}$ for $d \simeq 160,110$ and $50 \mathrm{~nm}$, respectively. The Néel temperature $T_{N}$ was extracted from the reduced temperature dependence of the magnetic moment, $M_{F C} / M_{\mathrm{ZFC}}(T)$, at $H=100 \mathrm{Oe}$ (shown as the inset in Fig. 2(a)) and essentially the same for all the films, $T_{N} \simeq 125 \mathrm{~K}$. The peak position for the thinner film or the well-defined kink for the thicker ones in the $M_{F C}$ l $M_{\text {ZFC }}(T)$ curve was associated with the onset of the AFM transition (indicated by an arrow). Therefore, the Curie temperature decreases rapidly with decreasing thickness of the NSMO film and falls to below the Néel point for $d \simeq 50 \mathrm{~nm}$.

The effect of lattice-strain on the Curie temperature of these films has been discussed in detail ${ }^{10}$ on the basis of the Millis model. ${ }^{11}$ It was found that the change in the crystal lattice parameters with decreasing thickness can lead to the observed suppression of spin ordering in these films.

Figure 2(b) shows the FC $M(T)$ curves for the same films, taken at $H=1 \mathrm{~T}$, which can be treated as the temperature variations in the saturated magnetic moment. This is 


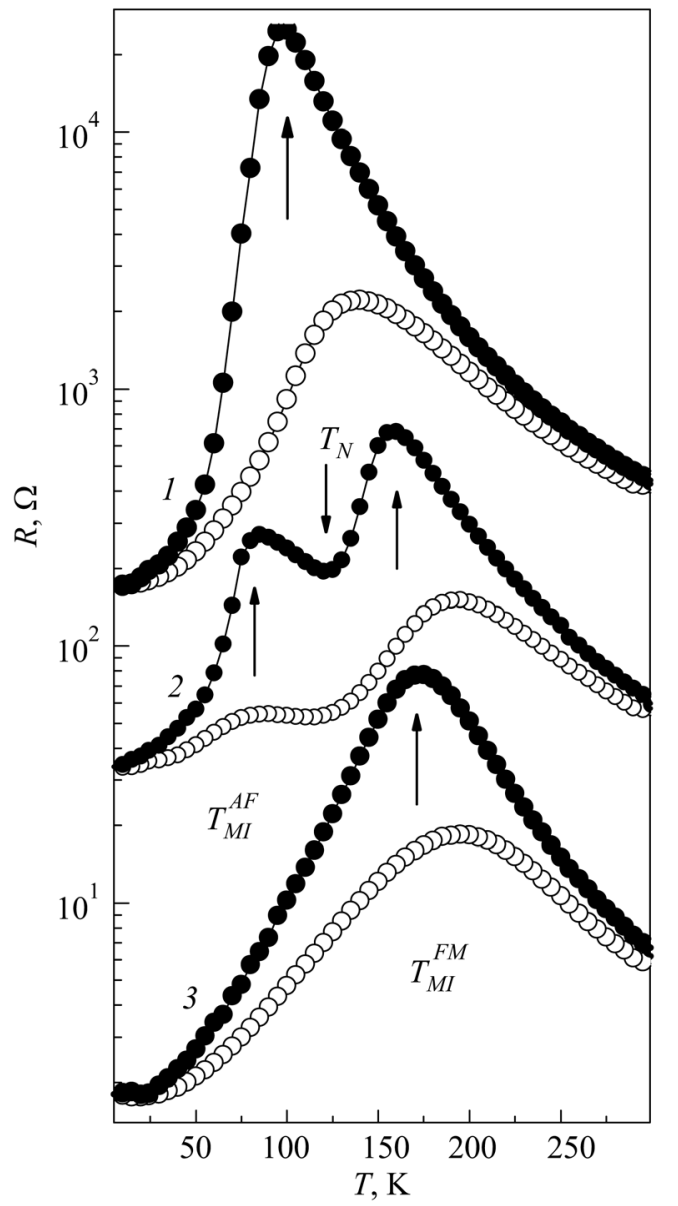

FIG. 3. Temperature dependence of the resistance for NSMO with $d=50$ (1), 110 (2), and 160 (3) nm without (solid symbols) and with (open symbols) an applied magnetic field of $5 \mathrm{~T}$. The MI transition temperature is indicated by the arrows. $T_{N}$ indicates the Néel point. The curves are only guides for the eye.

confirmed by the magnetic hysteresis loops for the same films, shown in the inset to Fig. 2(b), which manifests a saturation of the magnetic moment even in an applied magnetic field of $1 \mathrm{~T}$ and at a low temperature $(10 \mathrm{~K})$.

Figure 3 is the temperature-dependent resistance $R(T)$ for the NSMO films with $d \simeq 160,110$ and $50 \mathrm{~nm}$ without and with an applied magnetic field of $5 \mathrm{~T}$. It can be seen that the thicker and thinner films undergo a complete metal-insulator (MI) transition with decreasing temperature: $T_{M I} \simeq 175$ and $95 \mathrm{~K}$ for $d \simeq 160$ and $50 \mathrm{~nm}$, respectively. At the same time, the film with $d \simeq 110 \mathrm{~nm}$ manifests two MI transitions at 160 and $85 \mathrm{~K}$, which are sensitive to the applied magnetic field. This effect can not be explained by the existence of an additional FM phase with a different Curie point, because the FM $M(T)$ dependence for this film has only a single FM transition (see Fig. 2).

Figure 4 shows the temperature dependence of the magnetoresistance ratio for the same films. MR is defined as $100 \% \times[R(0)-R(H)] / R(H)$, where $R(H)$ and $R(0)$ are the resistances with and without, respectively, an applied magnetic field of $5 \mathrm{~T}$. The main peculiarity of the films studied here is an enormous increase in the CMR effect with decreasing thickness. Thus, $\mathrm{MR} \simeq 60000 \%$ for a thin film $(d \simeq 50 \mathrm{~nm})$ while it does not exceed $400 \%$ for a thick one $(d \simeq 160 \mathrm{~nm})$.

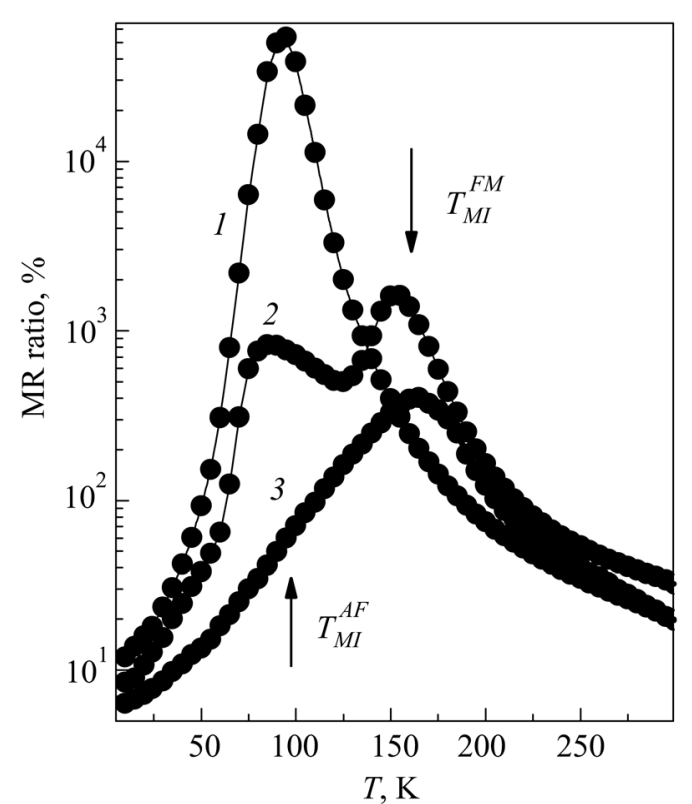

FIG. 4. Temperature dependence of the MR ratio for NSMO with $d=50$ (1), $110(2)$, and 160 (3) $\mathrm{nm}$. The curves are only guides for the eye.

\section{DISCUSSION}

The following intriguing results require discussion: (i) the appearance of a second MI transition with decreasing temperature in the intermediate thickness film $(d \simeq 110 \mathrm{~nm})$ and (ii) an enormous increase in the MR ratio in the thinnest film $(d \simeq 50 \mathrm{~nm})$.

An explanation for the observed peculiarities in $R(T)$ must account for the following experimental facts. First, because the FC $M(T)$ behavior is the same at the Néel temperature for all the films (see Fig. 2), one can conclude that the AFM phase arises from a precursor paramagnetic state rather than from the existing FM phase. Therefore, both the FM and AFM phases co-exist separately from one other in the film at low temperature $\left(T \leq T_{C}, T_{N}\right)$ and can be treated as a percolating net of metallic (FM) and insulating (AFM) resistors. This is the principal difference between the samples and the bulk materials ${ }^{6}$ or half-doped films. ${ }^{12}$ Second, using the magnetic hysteresis loops and the FC $M(T)$ curves for $H=1 \mathrm{~T}$ shown in Fig. 2(b), one can estimate the concentration of the FM (metal) phase in our films at different temperatures as $M_{s}(T) / M_{s}^{\text {bulk }}$, where $M_{s}^{\text {bulk }} \simeq 3.5 \mu_{B} / \mathrm{Mn}$ is the magnetic moment for the bulk NSMO in the fully FM state. ${ }^{13}$ At low temperatures $M_{s}(0) / M_{s}^{\text {bulk }} \simeq 0.49,0.77$, and 0.91 , while at the Néel point $M_{s}\left(T_{N}\right) / M_{s}^{\text {bulk }} \simeq 0.154,0.371$, and 0.591 for $d \simeq 50,110$ and $160 \mathrm{~nm}$, respectively. At the same time the percolation threshold for the 3-dimensional magnetic percolating system is $p_{c} \simeq 0.395 .{ }^{14-16}$ Consequently, two kinds of resistor circuit can be realized in the film depending on the concentration of the FM metal phase: a parallel-resistor circuit for $M_{s}(T) / M_{s}^{\text {bulk }} \geq p_{c}$ and a seriesconnected resistor for $M_{s}(T) / M_{s}^{\text {bulk }} \leq p_{c}$, where the FM and AFM phases play the roles of separated resistors with a different temperature behaviors.

Given the above, we suggest the following explanation for the unusual behavior of $R(T)$ in the NSMO films. Thick films $(d \simeq 160 \mathrm{~nm})$ undergo a classical MI transition at $T_{M I}^{F M} \simeq 175 \mathrm{~K}$ through the regular DE mechanism, because 
$M_{S}(T) / M_{s}^{\text {bulk }} \geq p_{c}$ over the entire temperature range and the parallel-resistor circuit, alone, is realized. Therefore, the low-resistance FM metal-like phase shunts the high-resistance AFM phase, which exists for $T_{N} \ll T_{C}$, and the corresponding $R(T)$ rise with decreasing temperature associated with the CO AFM state, does not show up in the experimental curve. A similar transition occurs in the intermediate film with $d \simeq 110 \mathrm{~nm}$ at high temperature, $T_{M I}^{F M} \simeq 160 \mathrm{~K}$ above $T_{N}$ and not very far from the Curie point, $T_{C} \simeq 180 \mathrm{~K}$. At the same time, the concentration of the $\mathrm{FM}$ phase $(\simeq 0.371)$ does not exceed the percolation threshold at the Néel point, thereby providing the series-resistor circuit between the FM and AFM phases. Figure 3 shows that the resistance sharply increases for $T \leq T_{N}$, as is typical for this compound and explained by electron localization in the CO AFM state. ${ }^{6,12}$ However, in our case, the expected increase in $R(T)$ with decreasing temperature does not extend to low temperatures and the resistance begins to fall off below a certain temperature, thereby forming the additional peak in $R(T)$ $T_{M I}^{A F} \simeq 85 \mathrm{~K}$. At this temperature the concentration of the FM phase $(\simeq 0.57)$ exceeds the percolation threshold and forms an infinitely conducting cluster which shunts the $\mathrm{CO}$ AFM resistance. In contrast to the thicker films, a thin NSMO film $(d \simeq 50 \mathrm{~nm})$ undergoes an AFM transition above the FM transition. Therefore, the observed rise in resistance for $T \leq T_{N}$ with decreasing temperature only results from formation of the CO AFM state. On further cooling the FM phase appears in the film and completely shunts the AFM phase at a low temperature, $T \leq T_{M I}^{A F} \simeq 95 \mathrm{~K}$, thereby yielding the metal-like behavior of $R(T)$.

The observed large difference in the MR ratio between thick and thin films is related to different mechanisms for the MI transition. In the first case, the FM metal-like state develops from the paramagnetic (PM) insulating phase by the DE mechanism. An applied magnetic field increases the Curie point and shifts the MI transition to higher temperatures, causing a CMR effect. In the second case, a PM $\rightarrow$ AFM transition takes place in the film and the FM phase acts as a shunt-resistor. In this case the MI transition has a percolation origin and is caused by a competition between the FM metal-like and the AFM insulating phase concentrations. A similar mechanism for CMR has been proposed recently, which includes stabilization of the CO AFM state among correlated polaron clusters owing to AFM coupling. ${ }^{2}$ The huge drop of the resistance in an applied magnetic field, in that case, is governed by a collapse (melting) of the $\mathrm{CO}$ state, which leads to an enhanced CMR effect. ${ }^{13}$

\section{CONCLUSIONS}

We have shown that the magnetic- and electron-phase states are controlled by the thickness of $\mathrm{Nd}_{0.52} \mathrm{Sr}_{0.48} \mathrm{MnO}_{3}$ films. The thickest film in these experiments manifests almost complete FM ordering and the MI transition is realized through the regular DE mechanism. The film with an intermediate thickness manifests an additional MI transition with decreasing temperature, which is provided by competition between the increasing FM phase and the AFM ordering, and has a percolation origin. The thinnest film has an MR ratio of up to $\simeq 60000 \%$ for an applied magnetic field of $5 \mathrm{~T}$. The observed enhanced CMR effect in the thinnest film is explained by formation of a $\mathrm{CO}$ AFM state at a temperature above the Curie point and the collapse of the CO state in the high magnetic field. Thus, the deposition technique enables us to control the mechanism of the CMR effect in thin $\mathrm{Nd}_{0.52} \mathrm{Sr}_{0.48} \mathrm{MnO}_{3}$ films. This is a first step toward strongly correlated electron devices.

This work was supported by the NRF/MEST through the Quantum Photonic Science Research Center, Korea. V. Svetchnikov is grateful to the financial support of Netherlands Institute for Metal Research.

a)Email: pvg@imp.kiev.ua

${ }^{1}$ For a review, see Colossal Magnetoresistance, Charge Ordering and Related Properties of Manganese Oxides, edited by C. N. R. Rao and B. Raveau (World Scientific, Singapore, 1998); Colossal Magnetoresistance Oxides, edited by Y. Tokura (Gordon and Breach, London, 1999); E. Dagotto, T. Hotta, and A. Moreo, Phys. Rep. 344, 1 (2001).

${ }^{2}$ C. S en, G. Alvarez, and E. Dagotto, Phys. Rev. Lett. 98, 127202 (2007).

${ }^{3}$ V. G. Prokhorov, G. G. Kaminsky, J. M. Kim, T. W. Eom, J. S. Park, Y. P. Lee, V. L. Svetchnikov, G. G. Levtchenko, A. V. Paschenko, Yu. V. Medvedev, Yu. M. Nikolaenko, G. V. Bukin, and V. A. Khokhlov, Fiz. Nizk. Temp. 37, 141 (2011) [Low Temp. Phys. 37, 112 (2011)].

${ }^{4}$ V. N. Varyukhin, Yu. V. Medvedev, Yu. M. Nikolaenko, A. B. Mukhin, B. V. Belyaev, V. A. Gritskikh, I. V. Zhikharev, S. V. Kara-Murza, N. V. Korchikova, and A. A. Tikhii, Tech. Phys. Lett. 35, 937 (2009).

${ }^{5}$ R. Kajimoto, H. Yoshizawa, H. Kawano, H. Kuwahara, Y. Tokura, K. Ohoyama, and M. Ohashi, Phys. Rev. B 60, 6506 (1999).

${ }^{6} \mathrm{Z}$. Q. Li, H. Liu, Y. H. Cheng, W. B. Mi, A. Yu, H. L. Bai, and E. I. Jiang, Physica B 353, 324 (2004).

${ }^{7}$ W. Prellier, A. Biswas, M. Rajeswari, T. Venkatesan, and R. L. Greene, Appl. Phys. Lett. 75, 397 (1999).

${ }^{8}$ Y. H. Hyun, S. Y. Park, Y. P. Lee, V. G. Prokhorov, and V. L. Svetchnikov, Appl. Phys. Lett. 91, 262505 (2007).

${ }^{9}$ V. G. Prokhorov, G. G. Kaminsky, V. A. Komashko, Y. P. Lee, S. Y. Park, Y. H. Hyun, J. B. Kim, J. S. Park, V. L. Svetchnikov, V. P. Pashchenko, and V. A. Khokhlov, Fiz. Nizk. Temp. 33, 889 (2007) [Low Temp. Phys. 33, 678 (2007)].

${ }^{10}$ V. G. Prokhorov, Y. H. Hyun, J. S. Park, J. B. Kim, G. H. Kim, Y. S. Lee, Y. P. Lee, and V. L. Svetchnikov, J. Appl. Phys. 104, 103901 (2008).

${ }^{11}$ A. J. Millis, T. Darling, and A. Migliori, J. Appl. Phys. 83, 1588 (1998).

${ }^{12}$ Y. Ogimoto, M. Nakamura, N. Takubo, H. Tamaru, M. Izumi, and K. Miyano, Phys. Rev. B 71, 060403 (2005).

${ }^{13}$ J. Geck, D. Buns, C. Hess, R. Klingeler, P. Reutler, M. v. Zimmermann, S.-W. Cheong, and B. Büchner, Phys. Rev. B 66, 184407 (2002).

${ }^{14}$ Y. Xiong, S.-Q. Shen, and X. C. Xie, Phys. Rev. B 63, 140418 (2001).

${ }^{15}$ V. G. Prokhorov, G. G. Kaminsky, V. A. Komashko, Y. P. Lee, and J. S. Park, Fiz. Nizk. Temp. 29, 885 (2003) [Low Temp. Phys. 29, 663 (2003)].

${ }^{16}$ V. G. Prokhorov, V. S. Flis, G. G. Kaminsky, Y. P. Lee, J. S. Park, and V. L. Svetchnikov, Fiz. Nizk. Temp. 31, 213 (2005) [Low Temp. Phys. 31, $161(2005)]$.

This article was published in English in the original Russian journal. Reproduced here with stylistic changes by AIP. 